\title{
RELATIONS AMONG SMOOTH KERVAIRE CLASSES AND SMOOTH INVOLUTIONS ON HOMOTOPY SPHERES
}

\author{
By YasuHiKo KitadA
}

\section{$\S 1$. Introduction and statement of results.}

In [9], Rourke and Sullivan defined classes $K_{4 \imath+2} \in H^{4 \imath+2}(G / P L, \boldsymbol{Z} / 2)$ that are characterized by the property that for a normal map $f: M \rightarrow G / P L$ of a closed $P L$ manifold $M$, the Kervaire obstruction of $f$ is given by the formula

$$
c(f)=\left\langle V(M)^{2} f^{*}\left(\sum_{\imath} K_{4 \imath+2}\right),[M]\right\rangle,
$$

where $V(M)=1+v_{1}(M)+v_{2}(M)+\cdots$ is the total $W u$ class of $M$.

Let $k_{4 \imath+2} \in H^{4 \imath+2}(G / O, \boldsymbol{Z} / 2)$ be the image of $K_{4 \imath+2}$ by the map $H^{*}(G / P L, \boldsymbol{Z} / 2)$ $\rightarrow H^{*}(G / O, \boldsymbol{Z} / 2)$ which is induced by the natural map $G / O \rightarrow G / P L$. We shall call $k_{42+2}$ the smooth Kervaire class. The work of Brumfiel, Madsen and Milgram [1] gives fundamental properties concerning the smooth Kervaire classes.

In this paper we shall first prove the following theorem which gives an interesting relation between two smooth Kervaire classes in different dimensions.

THEOREM A. Let $r$ and $s$ be integers that satisfy $r>s \geqq 2$. Then we have the relation

$$
S q^{2^{r-1}} S q^{2^{r-2}} \cdots S q^{2^{s-1}} S q^{2^{s-2}} k_{2 s-2}=S q^{2^{s-1}} S q^{2^{s-2}} k_{2 r-2} .
$$

The formula (1.1) involves Kervaire classes of various degrees. So one might hope to obtain some information about the Kervaire obstructions for certain manifolds in the smooth category if one uses this relation. In fact, as an application of Theorem A, we can show

THEOREM B. Let $n$ be a positive integer such that $n+1$ is not a fower of 2 and let $m$ be a non-negative integer with $m \leqq 2 n$. Then the Kervarre obstruction map

$$
c:\left[S^{2 m+1} \times P^{4 n-2 m+1}, G / O\right] \rightarrow Z / 2
$$

is trivial.

Received February 25, 1988 
Theorem B has a geometric meaning which we shall now explain. Let $f: M^{n} \rightarrow N^{n}$ be an equivariant map between smooth manifolds with involutions. We shall say that $f$ is $t$-isovariant if $f$ is isovariant (i.e. $f$ preserves the isotropy groups at each point) and in addition if $f$ can be regarded as an equivariant vector bundle map inducing a linear isomorphism in each fiber of the normal bundle around the fixed point sets. A smooth involution on a homotopy sphere is called almost linear if the fixed point set is diffeomorphic to the standard sphere. Theorem B implies that the surgery obstruction map

$$
c:\left[D^{2 m+1} \times P^{4 n-2 m+1} / S^{2 m} \times P^{4 n-2 m+1}, G / O\right] \rightarrow \boldsymbol{Z} / 2
$$

is trivial. Then using the surgery theory with $\pi_{1}=\boldsymbol{Z} / 2$, we obtain the following corollary.

COROLlaRY C. If $n+1$ is not a power of 2 then there does not exist an orientation reversing almost linear smooth involution on the Kervaire sphere of dimension $4 n+1$ which is t-isovariantly homotopy equivalent to a linear involution on the standard sphere.

In brief, Corollary $\mathrm{C}$ means that an orientation reversing involution on the Kervaire sphere does not admit a "good" isovariant linear model. We shall consider now two special cases. The first one is the involution $\left(W_{3}^{4 n+1}, T_{3}\right)$ studied in [3]. According to Corollary $\mathrm{C}$ above, this involution is not $t$-isovariantly homotopy equivalent to a linear involution $L$ on the standard sphere $S^{4 n+1}$ with $\operatorname{Fix}(L)=S^{2 n}$ although it is equivariantly homotopy equivalent to the linear involution $\left(S^{4 n+1}, L\right)$ and more than that it is even equivariantly normally cobordant to the linear involution $\left(S^{4 n+1}, L\right)$. To be precise, there arises a homotopical obstruction $\left[\iota_{2 n+1}, \iota_{2 n+1}\right]$ to the existence of a $t$-isovariant map from $\left(W_{3}^{4 n+1}, T_{3}\right)$ to $\left(S^{4 n+1}, L\right)$. The second interesting case arises when $m=0$, that is, when the dimension of the fixed point set is zero. In this case (1.2) says that the obstruction map

$$
c:\left[P^{4 n+1} \times I / P^{4 n+1} \times \partial I, G / O\right] \rightarrow Z / 2
$$

is zero. From the surgery exact sequence of Wall [11], Theorem 10.8, this is equivalent to saying that the action of the Wall group $L_{4 n+2}(\boldsymbol{Z} / 2,+) \cong \boldsymbol{Z} / 2$ on the set of equivalence classes of homotopy smoothings $h S\left(P^{4 n+1}\right)$ is non-trivial. This action corresponds to taking the connected sum of $P^{4 n+1}$ with the homotopy spheres bounding parallelizable manifolds and making an appropriate homotopy equivalence to $P^{4 n+1}$. But since any orientation preserving self homotopy equivalence of $P^{4 n+1}$ is homotopic to the identity, we do not have to consider the homotopy classes of maps to $P^{4 n+1}$ in homotopy smoothings. Thus we have obtained

COROLLARY D. The connected sum of the real projective space $P^{4 n+1}$ with the Kervaire sphere is not diffeomorphic to $P^{4 n+1}$ when $4 n+4$ is not a power of 2 . 
Corollary D gives an answer to the problem of [2], p. 427, originally due to W. Browder (see also [4], p. 95). We shall now state the notations which will be used in this paper. All homology and cohomology coefficients are $\boldsymbol{Z} / 2$ and binomial coefficients are considered $\bmod 2$. Let $Q\left(S^{0}\right)=\lim _{n \rightarrow \infty} \Omega^{n} S^{n}$ and let $S G=\left(Q\left(S^{0}\right)\right)_{1}$ be the subspace of degree 1 maps $S^{\infty} \rightarrow S^{\infty}$. The natural map $i: S G \rightarrow G / O$ induces an epimorphism in homology [7] and hence a monomorphism in cohomology. So we may identify $H^{*}(G / O)$ with a subalgebra of $H^{*}(S G) . Q\left(S^{0}\right)$ is an infinite loop space with respect to the loop sum operation denoted by $*$ and let $Q^{2}$ be the Dyer-Lashof homology operation for the loop sum. $G / O$ is an infinite loop space whose $H$-space structure corresponds to the Whitney sum operation. The map $i$ above is an infinite loop map for the composition operation on $S G$. For details on these facts, see [1], [5], [6] and [7].

The program of this paper is as follows: in $\S 2$ we shall recall some basic facts of the cohomology and homology of $S G$ and develop some additional properties which will be used later. In $\S 3$, we shall prove Theorem $A$ which requires a manipulation of mod 2 binomial coefficients. In $\S 4$, we shall show how Theorem A can be applied to the triviality of the obstruction map stated in Theorem B.

\section{§2. Homology and Steenrod operations on $H_{*}(S G)$.}

Let $I=\left(\imath_{1}, i_{2}, \cdots, \imath_{n}\right)$ be a sequence of non-negative integers. We shall write $Q^{I}$ or sometimes $Q(I)$ in place of the operation $Q^{\imath_{1}} \cdots Q^{\imath_{n}}$. We say that $I$ or $Q^{I}$ is allowable if $i_{\jmath} \leqq 2 \imath_{\jmath_{+1}}$ holds for $j=1, \cdots, n-1$. The length of $I, l(I)$ is the number of integers contained in $I$ and we define its excess $e(I)$ to be

$$
\sum_{j=1}^{n-1}\left(\imath_{j}-2 i_{j+1}\right)+\imath_{n}=\imath_{1}-\imath_{2}-\cdots-i_{n} .
$$

As to the Pontrjagin ring structure of $S G$, the following result is well known.

Theorem 2.1 ([6], [7]). The Pontrjagin ring of $S G$ with respect to the composition product is given by

$$
\begin{aligned}
H_{*}(S G)= & E\left\{Q^{i}[1] *[-1] \mid i \geqq 1\right\} \otimes P\left\{Q^{\imath} Q^{i}[1] *[-3] \mid i \geqq 1\right\} \\
& \otimes P\left\{Q^{I}[1] *\left[1-2^{n}\right] \mid I \text { allowable, } l(I)=n \geqq 2, e(I) \geqq 1, i_{n} \geqq 1\right\},
\end{aligned}
$$

where $E$ (resp. $P$ ) is an exterior (resp. polynomal) algebra over $\boldsymbol{Z} / 2$ on given generators.

We shall denote the element $Q^{2}[1] *[-1]$ by $e_{\imath}$ which coincides with the image of $P^{2}$ by the natural map

$$
P^{\imath} \longrightarrow S O(i+1) \longrightarrow S O \longrightarrow S G .
$$


Since the natural map $i_{*}: H_{*}(S G) \rightarrow H_{*}(G / O)$ is surjective ([7]), the dual map $i^{*}: H^{*}(G / O) \rightarrow H^{*}(S G)$ is injective. So we may identify $k_{4 \imath+2}$ with its image $i^{*}\left(k_{4 \imath+2}\right)$. have

Let $\psi: H_{*}\left(Q\left(S^{0}\right)\right) \rightarrow H_{*}\left(Q\left(S^{0}\right)\right) \otimes H_{*}\left(Q\left(S^{0}\right)\right)$ be the diagonal map. Then we

$$
x \cdot(y * z)=\sum_{\imath} x_{\imath}^{\prime} y * x_{\imath}^{\prime \prime} y
$$

where $\phi(x)=\sum_{\imath} x_{i}^{\prime} \otimes x_{\imath}^{\prime \prime}$ ([7]). Here the dot "." stands for the composition product which is often omitted.

The following formulas will be used in what follows: (May's formula [5])

$$
Q^{a}(x) y=\sum_{t} Q^{a+t}\left(x \cdot S q_{*}^{t}(y)\right),
$$

where $S q_{*}^{t}$ is the Kronecker dual of the Steenrod square.

(Dual May's formula [5])

$$
Q^{a}(x)=\sum_{t} Q^{a+t}[1] \cdot \chi\left(S q^{t}\right)_{*} x,
$$

where $\chi$ is the canonical anti-automorphism of the Steenrod algebra ([10], II $§ 4)$. (Cartan formula)

$$
Q^{2}(x * y)=\sum_{t} Q^{t}(x) * Q^{\imath-t}(y)
$$

(Adem relation)

$$
Q^{a} Q^{b}(x)=\sum_{t}\left(\begin{array}{c}
t-b-1 \\
2 t-a
\end{array}\right) Q^{a+b-t} Q^{t}(x) .
$$

(Nishida relation [8])

$$
S q_{*}^{a} Q^{b}(x)=\sum_{t}\left(\begin{array}{c}
b-a \\
a-2 t
\end{array}\right) Q^{b-a+t} S q_{*}^{t}(x)
$$

Let $*: S G \times S G \rightarrow S G$ be the $H$-space structure defined by $(x, y) \rightarrow x * y *[-1]$ used in [1], where $[-1]$ denotes a fixed map of degree -1 considered as an element of $Q\left(S^{0}\right)$. This defines a product

$$
\text { * : } H_{*}(S G) \otimes H_{*}(S G) \longrightarrow H_{*}(S G)
$$

and a cohomology coproduct

$$
\psi^{*}: H^{*}(S G) \longrightarrow H^{*}(S G) \otimes H^{*}(S G) .
$$

The following theorem is one of the main results of [1].

THEOREM 2.2. (1) $k_{4 \imath+2}=0$ unless $4 i+4$ is a power of 2 .

(2) If $n=2^{j}-2$ then 


$$
\psi^{*}\left(k_{n}\right)=k_{n} \otimes 1+1 \otimes k_{n}+\sum \sigma\left(w_{s+1}\right) \otimes \sigma\left(w_{t+1}\right),
$$

where the summation runs over $s+t=n, s, t>0$ and $\sigma\left(w_{\imath}\right)$ is the homology suspension of the Strefel-Whitney class $w_{i} \in H^{i}(B S G)$.

(3) $\sigma\left(w_{\imath}\right)$ is primitive for both composition and loop products.

(4) $\left\langle k_{2 \imath-2}, e_{a} * e_{b}\right\rangle=1$ if and only if $a+b=2^{\imath}-2$ and $\left\langle k_{2 \imath-2}, e_{\imath_{1}} * \cdots \underline{*} e_{\imath_{n}}\right\rangle=0$ if $n \geqq 3$.

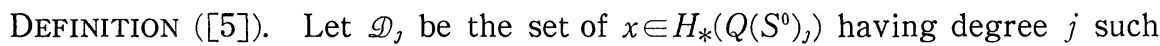
that $x *[1-j]$ is composition decomposable in $H_{*}(S G)$. Put $\mathscr{D}=\bigoplus_{\jmath} \mathscr{D}_{\jmath}$. Let $\mathscr{g}$ denote the ideal of positive dimensional elements of $H_{*}\left(Q\left(S^{0}\right)\right)$.

THEOREM 2.3 (Madsen [4]).

(1) $\mathscr{I}_{*} \mathscr{I}_{*} \mathscr{I} \subset \mathscr{D}, \quad\left(I_{*} \mathscr{I}\right) \cdot \mathscr{I} \subset \mathcal{D}$ and

(2) $\quad Q^{a}(\mathcal{I} * \mathscr{G}) \subset \mathscr{D}, \quad Q^{a}(\mathscr{D}) \subset \mathscr{D}$.

As an easy application of (2.1), given $x, y \in H^{*}(S G)$, we have

$$
\begin{aligned}
& \{(x *[1]) \cdot(y *[1])\} *[-3]=x \cdot y+x \underline{*} y+\sum x y_{j}^{\prime} * y_{j}^{\prime \prime}+\sum x_{\imath}^{\prime} y * x_{\imath}^{\prime \prime} \\
& +\sum x \underline{*} y_{j}^{\prime} y_{j}^{\prime \prime}+\sum x_{i}^{\prime} \underline{x} x_{\imath}^{\prime \prime} \underline{y}+\sum x_{\imath}^{\prime} y_{j}^{\prime} x_{\imath}^{\prime \prime} \underline{*} y_{j}^{\prime \prime},
\end{aligned}
$$

where

$$
\begin{aligned}
& \psi(x)=x \otimes[1]+[1] \otimes x+\sum x_{i}^{\prime} \otimes x_{\imath}^{\prime \prime} \quad \text { and } \\
& \psi(y)=y \otimes[1]+[1] \otimes y+\sum y_{j}^{\prime} \otimes y_{j}^{\prime \prime} .
\end{aligned}
$$

Since $\mathscr{I}_{*} \mathscr{I}_{*} \mathscr{I} \subset \mathscr{D}$ by Theorem 2.3 , we have

LEMMA 2.4. Let $x, y \in H_{*}(S G)$ then

$$
\{(x *[1]) \cdot(y *[1])\} *[-3] \equiv x \underline{x}+\sum x_{\imath}^{\prime} y * x_{\imath}^{\prime \prime}+\sum x y_{j}^{\prime} y_{j}^{\prime \prime} \quad \bmod \mathscr{D}_{1} .
$$

We shall next determine the values of $k_{2 \imath-2}$ on the elements that include the algebra generators of $H_{*}(S G)$.

LEMMA 2.5. Let $a, b$ be positvve integers with $a+b=2^{r}-2$ for some $r$. Then we have

$$
\left\langle k_{2 r-2},\left(Q^{a}[1] \cdot Q^{b}[1]\right) *[-3]\right\rangle=1 .
$$

Proof. Let $x=e_{a}=Q^{a}[1] *[-1], y=e_{b}=Q^{b}[1] *[-1]$ and apply Lemma 2.4, then we have

$$
\left(Q^{a}[1] \cdot Q^{b}[1]\right) *[-3] \equiv e_{a} * e_{b}+\sum_{0<i<a} e_{i} e_{b} * e_{a-i}+\sum_{0<j<b} e_{a} e_{j} * e_{b-\jmath}
$$

$\bmod \mathscr{D}_{1}$. By the primitivity of the smooth Kervaire classes ([1], Lemma 3.2), $k_{2 r-2}$ vanishes on $\mathscr{D}_{1}$. It also vanishes on $e_{\alpha} e_{\beta} * e_{\gamma}$ by Theorem 2.2 (2) and (3). Therefore by Theorem 2.2 (4) we get the assertion. 
LEMMA 2.6. $\chi\left(S q^{a}\right)_{*} Q^{b}[1]=\left(\begin{array}{c}a+b \\ a\end{array}\right) Q^{b-a}[1]$.

Proof. We may put $\chi(S q)_{*} Q^{b}[1]=\sum_{t} \varepsilon_{t} Q^{b-t}[1]$, where $\varepsilon_{t} \in \boldsymbol{Z} / 2$ and $S q=$ $1+S q^{1}+S q^{2}+\cdots$. Then from the Nishida relation (2.6), we have

$$
\begin{aligned}
Q^{b}[1] & =S q_{*} \sum_{t} \varepsilon_{t} Q^{b-t}[1]=\sum_{t, s} \varepsilon_{t} S q_{*}^{s} Q^{b-t}[1] \\
& =\sum_{t, s} \varepsilon_{t}\left(\begin{array}{c}
b-t-s \\
s
\end{array}\right) Q^{b-t-s}[1] \\
& =\sum_{m \leqq b} \sum_{s \leqq m}\left(\begin{array}{c}
b-m \\
s
\end{array}\right) \varepsilon_{t} Q^{b-m}[1] .
\end{aligned}
$$

This gives us a system of equations for $\varepsilon_{t}$ :

$$
\sum_{s=0}^{m}\left(\begin{array}{c}
b-m \\
s
\end{array}\right) \boldsymbol{\varepsilon}_{m-s}= \begin{cases}1 & \text { if } m=0 \\
0 & \text { if } m=1, \cdots, b .\end{cases}
$$

This linear equation has a unique solution since the matrix of coefficients is triangular with non-zero diagonal entries. We can easily check that the solution is given by $\varepsilon_{t}=\left(\begin{array}{c}b+t \\ t\end{array}\right)$, which can be verified by the equality $([6], 6.20)$ :

$$
\sum_{i=0}^{c}\left(\begin{array}{c}
a \\
c-i
\end{array}\right)\left(\begin{array}{c}
b+\imath \\
i
\end{array}\right)=\left\{\begin{array}{cc}
\left(\begin{array}{c}
a-b-1 \\
c
\end{array}\right) & (\text { if } a>b) \\
\left(\begin{array}{c}
b+c-a \\
c
\end{array}\right) & (\text { if } a \leqq b) .
\end{array}\right.
$$

LEMMA 2.7. Let $Q^{a} Q^{b}$ be allowable and $a, b>0$. Then

$$
\left\langle k_{2 r-2}, Q^{a} Q^{b}[1] *[-3]\right\rangle=1
$$

holds if and only if $a=b=2^{r-1}-1$. have

Proof. We may assume that $a+b=2^{r}-2$. By (2.3) and Lemma 2.6, we

$$
Q^{a} Q^{b}[1]=\sum_{\imath=0}^{b}\left(\begin{array}{c}
b+\imath \\
\imath
\end{array}\right) Q^{a+i}[1] \cdot Q^{b-i}[1]
$$

Let $\left[\begin{array}{l}\alpha \\ \beta\end{array}\right]$ denote the coefficient of $t^{\beta}$ in the formal power series $(1+t)^{\alpha} \in \boldsymbol{Z} / 2[[t]]$. It is an easy exercise to see that $\left(\begin{array}{l}\alpha \\ \beta\end{array}\right)=\left[\begin{array}{c}\beta-\alpha-1 \\ \beta\end{array}\right]$ when $\alpha \geqq \beta \geqq 0$. Hence by Lemma 2.5, we have

$$
\begin{aligned}
& \left\langle k_{2 r-2}, Q^{a} Q^{b}[1] *[-3]\right\rangle=\sum_{i=0}^{b}\left(\begin{array}{c}
b+i \\
b
\end{array}\right)=\sum_{i=0}^{b}\left[\begin{array}{c}
-b-1 \\
i
\end{array}\right] \\
& =\text { sum of the coefficients of } 1, t, \cdots, t^{b} \text { in }(1+t)^{-b-1}
\end{aligned}
$$




$$
=\left[\begin{array}{c}
-b-2 \\
b
\end{array}\right]=\left(\begin{array}{c}
2 b+1 \\
b
\end{array}\right) \text {. }
$$

This is nonzero mod 2 if and only if $b+1$ is a power of 2 (see Lemma 3.1). By allowability of $I=(a, b)$, we have $b \leqq a \leqq 2 b$. Hence the above expression is nonzero if and only if $b=2^{r-1}-1$.

PROPOSITION 2.8. The values of $k_{2 r_{-2}}$ on the algebra generators in Theorem 2.1 are given as follows:

(1) $\left\langle k_{2 r-2}, Q^{i}[1] *[-1]\right\rangle=0$,

(2) $\left\langle k_{2 r-2}, Q^{i} Q^{i}[1] *[-3]\right\rangle=1$ if and only if $i=2^{r-1}-1$, and

(3) Let $I=\left(i_{1}, \cdots, i_{n}\right)$ be allowable, then $\left\langle k_{2 r_{-2}}, Q^{I}[1] *\left[1-2^{n}\right]\right\rangle=0$ if $\left.n\right\rangle 2$ or $n=2$ and $e(I)>0$.

Proof. (1) is immediate since $Q^{i}[1] *[-1]$ comes from an element of $H_{*}(S O)$. We have already shown (2) in Lemma 2.7. We have also shown (3) in case $n=l(I)=2$ in Lemma 2.7. So we assume that $n \geqq 3$. We start with the case $n=3$. As we have seen in the proofs of Lemma 2.5 and Lemma 2.7, $Q^{b} Q^{c}[1]$ belongs to $\mathscr{I}_{*} \mathscr{I} \bmod \mathscr{D}$. From Theorem $2.3(2), Q^{a} Q^{b} Q^{c}[1]$ belongs to $\mathscr{D}$. Thus by induction on $n$, we can prove that $Q^{I}[1] \in \mathscr{D}$ if $l(I) \geqq 3$. Since $k_{2 r-2}$ vanishes on composition decomposables, the assertion follows.

Note that in Proposition 2.8, (3) we did not require $e(I)>0$ when $n>2$.

\section{§3. Proof of Theorem A.}

Before we begin the proof, we shall make a short study on $\bmod 2$ binomial coefficients. By the mod 2 binomial coefficient $\left(\begin{array}{l}a \\ b\end{array}\right)$ we mean the coefficient of $t^{b}$ of the polynomial $(1+t)^{a} \in \boldsymbol{Z} / 2[t]$. If either $a$ or $b$ is negative we set $\left(\begin{array}{l}a \\ b\end{array}\right)=0$ by convention. Let $a$ be a non-negative integer. Then $a$ has a unique expression $a=\sum_{i \geq 0} a_{i} 2^{2}$, where $a_{\imath}=0$ or 1 . We shall call this expression the 2-adic expansion of $a$.

LEMMA 3.1 ([10], I.2.6). Let $a=\sum a_{i} 2^{2}$ and $b=\sum b_{i} 2^{2}$ be the 2-adic expansions of $a$ and $b$. Then we have

$$
\left(\begin{array}{l}
a \\
b
\end{array}\right)=\Pi_{i}\left(\begin{array}{l}
a_{i} \\
b_{i}
\end{array}\right)
$$

In other words, for non-negative integers $a$ and $b$, we have $\left(\begin{array}{l}a \\ b\end{array}\right)=0$ if and only if there exists $i$ such that $a_{\imath}=0$ and $b_{i}=1$.

The following property is immediate, but is useful in calculations. 
LEMMA 3.2. The mod 2 binomial coefficients are characterized by the following properties:

$\left(\begin{array}{l}0 \\ 0\end{array}\right)=1, \quad\left(\begin{array}{l}2 a \\ 2 b\end{array}\right)=\left(\begin{array}{l}a \\ b\end{array}\right), \quad\left(\begin{array}{c}2 a \\ 2 b+1\end{array}\right)=0, \quad\left(\begin{array}{c}2 a+1 \\ 2 b\end{array}\right)=\left(\begin{array}{l}a \\ b\end{array}\right) \quad$ and $\quad\left(\begin{array}{l}2 a+1 \\ 2 b+1\end{array}\right)=\left(\begin{array}{l}a \\ b\end{array}\right)$.

Using Lemma 3.2 we can prove the following lemma by induction on $n$.

LEMMA 3.3. (1) $\left(\begin{array}{c}2^{n}-1+i \\ 2 i\end{array}\right)$ is nonzero if and only if $i=2^{n}-2^{3}$ for some $j$ $(0 \leqq j \leqq n)$.

(2) $\left(\begin{array}{c}i \\ 2^{n}-2-i\end{array}\right)$ is nonzero of and only if $\imath=2^{n}-2^{\jmath}-1$ for some $j(0 \leqq j \leqq n-1)$.

Let $\left\{I_{j}\right\}_{j=1, \ldots, r}$ be a set of $n$-tuples $I_{J}=\left(i_{1}, \cdots, i_{n}\right)$ of positive integers not necessarily allowable. Then by repeated use of (2.5) we can express the sum $\sum_{j} Q\left(I_{j}\right)[1]$ as a sum of allowable terms as in $\sum_{k} Q\left(J_{k}\right)[1]$. In this case we say that $\sum Q\left(I_{j}\right)[1]$ contains (each of) $Q\left(J_{k}\right)[1]$. The proof of the following lemma is not difficult in principle, but requires a tedious arithmetic of $\bmod 2$ binomial coefficients.

LEMMA 3.4. Let $Q^{a} Q^{b}$ be allowable with $b>0$. If $r \geqq s \geqq 2$ then $S q_{*}^{2^{s-2}} S q_{*}^{2^{s-1}} Q^{a} Q^{b}[1]$ contains $Q^{2^{r-1}-1} Q^{2^{r-1}-1}[1]$ if and only if $a=2^{r-1}+2^{s-1}-1$ and $b=2^{r-1}+2^{s-2}-1$.

Proof. By counting degrees we must have $a+b=2^{r}+2^{s-1}+2^{s-2}-2$. So we may write $a=2^{r-1}+2^{s-1}-1+t$ and $b=2^{r-1}+2^{s-2}-1-t$ for some $t$. Then the allowability of $Q^{a} Q^{b}$ implies $3 t \leqq 2^{r-1}-1$. Using the Adem and Nishida relations we have

$$
S q_{*}^{2^{s-2}} S q_{*}^{2^{s-1}} Q^{a} Q^{b}[1]=\Sigma^{\prime} A B C D Q^{x} Q^{y}[1]+\Sigma^{\prime \prime} A B C D E Q^{2^{r-2-k}} Q^{k}[1],
$$

where

$$
\begin{aligned}
& A=\left(\begin{array}{c}
2^{r-1}-1+t \\
2^{s-1}-2 i
\end{array}\right), \quad B=\left(\begin{array}{c}
2^{r-1}+2^{s-2}-1-(t+i) \\
i
\end{array}\right), \\
& C=\left(\begin{array}{c}
2^{r-1}-2^{s-2}-1+t+\imath \\
2^{s-2}-2 j
\end{array}\right), \quad D=\left(\begin{array}{c}
2^{r-1}+2^{s-2}-1-(t+i+j) \\
j
\end{array}\right), \\
& E=\left(\begin{array}{c}
k-2^{r-1}-2^{s-2}+t+i+j \\
2 k-2^{r-1}+2^{s-2}+1-(t+i+j)
\end{array}\right), \\
& x=2^{r-1}-2^{s-2}-1+t+i+j \quad \text { and } y=2^{r-1}+2^{s-2}-1-(t+i+j) .
\end{aligned}
$$

The first summation $\Sigma^{\prime}$ runs over $i$ and $j$ satisfying $3(t+i+j) \leqq 2^{r-1}+2^{s-1}+2^{s-2}-1$ and $\Sigma^{\prime \prime}$ is over $i, j$ and $k$ such that $3(t+i+j) \geqq 2^{r-1}+2^{s-1}+2^{s-2}$ and $2 k \geqq 2^{r-1}$ $-2^{s-2}-1+t+i+j$. Here we may assume that $0 \leqq i \leqq 2^{s-2}$ and $0 \leqq j \leqq 2^{s-3}$ for otherwise $A$ or $C$ vanishes. For brevity of notation, we shall write $u$ in place 
of $Q^{2^{r-1}-1} Q^{2 r-1-1}[1]$. If we can show that $u$ is contained in $\Sigma^{\prime}+\Sigma^{\prime \prime}$ if and only if $t=0$, then the proof is complete. The proof is divided into several cases according to the values of $r$ and $s$.

Case $s=2$ : In this case we may assume that $j=0$ and $i=0$ or 1 . So in the second summation $\Sigma^{\prime \prime}$, we have $3 t \geqq 2^{r-1}$ which contradicts to the allowability condition. Therefore there are no terms in $\Sigma^{\prime \prime}$. Suppose $u$ is contained in $\Sigma^{\prime}$ as $A B C D Q^{x} Q^{y}[1]$, then we have $t+i+\jmath=2^{s-2}=1$, where $j=0$ and we see from Lemma 3.3 (1) that $A=\left(2^{r-1}-1+t\right)$ is nonzero if and only if $t=2^{r-1}-2^{q}$ for some $q \leqq r-1$. So if $r=2$, we must have $q=0, t=1$ or $q=1, t=0$. But the case $t=1$ contradicts to the allowability. Thus we must have $t=0$. When $r>2$, we have $t=2^{r-1}-2^{q}$ for some $q \leqq r-1$. If $q \leqq r-2$, we again get contradiction to the allowability. So we must have $q=r-1$ and $t=0$. Actually when $t=0, u$ is contained in $\Sigma^{\prime}$ for $i=1$. Hence $u$ is contained in $\Sigma^{\prime}$ if and only if $t=0$.

Case $s=3$ : When $r=s=3$, we can directly verify that $u$ is contained only in $S q_{*}^{1} S q_{*}^{2} Q^{7} Q^{5}[1]$. So we may assume that $r>3$. Suppose that $\Sigma^{\prime}$ contains $u$, for some $i$ and $j$, then we have $t+i+\jmath=2^{s-2}=2$. $C$ and $D$ are always nonzero for $0 \leqq j \leqq 1$. If $j=1$ then $B$ is nonzero if and only if $i=0$ and $t=1$. But then we have $A=\left(\begin{array}{c}2^{r-1} \\ 4\end{array}\right)=0$ since $r>3$. Therefore $u$ does not appear as a term with $j=1$. If $j=0$ then $B$ is nonzero and $A=\left(\frac{2^{r-1}-1+t}{2 t}\right)$ is nonzero if and only if $t=0$ from Lemma 3.3 (1) and the allowability condition. So $\Sigma^{\prime}$ contains $u$ if and only if $t=0$. We shall check if $u$ is contained in $\Sigma^{\prime \prime}$. If $u$ is contained in $\Sigma^{\prime \prime}$ for some $i, j$ and $k$, then we must have $k=2^{r-1}-1 . \quad E$ is nonzero if and only if $t+i+j-3=2^{r-1}-2^{q}-1 \quad(0 \leqq q \leqq r-2)$ by Lemma 3.3 (2). Then we have $t \geqq 2^{r-2}-1$, which contradicts to the allowability. So $\Sigma^{\prime \prime}$ does not contain $u$.

Case $r=s \geqq 4$ : Suppose $\Sigma^{\prime}$ contains $u$, then we have $t+i+j=2^{s-2}$. $D$ is always nonzero and $C=\left(\begin{array}{c}2^{s-1}-1-j \\ 2^{s-2}-2 j\end{array}\right)$ is nonzero if and only if $j=0$ or $2^{q}(q \leqq s-3)$ by a variation of Lemma $3.3(1)$. When $j=2^{q}$, we see that $B=\left(\begin{array}{c}2^{r-1}+2^{q}-1 \\ i\end{array}\right)$ is nonzero if and only if $0 \leqq \imath \leqq 2^{q}-1$. $A=\left(\begin{array}{c}2^{s-1}-1+t \\ 2^{q+1}+2 t\end{array}\right)$ is nonzero if and only if $t=2^{s-2}-2^{q}$ since $2^{s-2}-2^{q+1}+1 \leqq t \leqq 2^{s-2}-2^{q}$. If $q<s-3$ then we have $t \geqq 2^{s-3}+2^{s-4}$, which contradicts to the allowability. So we must have $q=s-3, t=2^{s-3}$ and $u$ is contained in $\Sigma^{\prime}$. On the other hand, when $j=0, B$ is nonzero and $A=\left(2^{s-1}-1+t\right)$ is nonzero if and only if $t=2^{s-1}-2^{c}(c \leqq s-1)$. By allowability we must have $c=s-1$ and $t=0$. Thus we have seen that $\Sigma^{\prime}$ contains $u$ if and only if $t=0$ or $2^{s-3}$. Next we turn to $\Sigma^{\prime \prime}$. If $\Sigma^{\prime \prime}$ contains $u$ for some $i, j$ and $k$, then we have $k=2^{s-1}-1$ and $E$ is nonzero if and only if $t+i+j=2^{s-1}+2^{s-2}-2^{q}$ $(0 \leqq q \leqq s-2)$ from Lemma 3.3 (2). Suppose $q<s-2$, then we have $t \geqq 2^{s-2}$ since we can assume that $\imath+\jmath \leqq 2^{s-2}+2^{s-3}$, but it contradicts to the allowability. Therefore we have $q=s-2$, that is, $t+\imath+\jmath=2^{s-1}$. Then $D$ is always nonzero 
and $C=\left(\begin{array}{c}2^{s-1}+2^{s-2}-1-j \\ 2^{s-2}-2 j\end{array}\right)$ is nonzero if and only if $j=2^{d}(d \leqq s-3) . \quad B$ is nonzero if and only if $0 \leqq i \leqq 2^{d}-1$ or $i=2^{s-2}$. However if $i \leqq 2^{d}-1$ then we have $t \geqq 2^{s-1}-2^{d+1}+1 \geqq 2^{s-2}$ and this contradicts to the allowability. If $i=2^{s-2}$ then we have $t=2^{s-2}-2^{d}$ and $d$ must be equal to $s-3$ since if $d<s-3$, we have $t \geqq 2^{s-3}+2^{s-4}$ contradicting to the allowability. When $d=s-3$, we have $t=2^{s-3}$ and $A$ is nonzero. Thus in this case, $\Sigma^{\prime \prime}$ contains $u$ if and only if $t=2^{s-3}$. But when $t=2^{s-3}$, as we have seen, $u$ is contained both in $\Sigma^{\prime}$ and in $\Sigma^{\prime \prime}$ canceling each other. Therefore $u$ appears in $\Sigma^{\prime}+\Sigma^{\prime \prime}$ if and only if $t=0$.

Case $r>s \geqq 4$ : We try to obtain the condition under which $\Sigma^{\prime}$ contains $u$. We have $t+i+j=2^{s-2}$. $\quad D$ is nonzero for any $j \leqq 2^{s-3}$. $\quad C=\left(\begin{array}{c}2^{r-1}-1-j \\ 2^{s-2}-2 j\end{array}\right)$ is nonzero if and only if $j=0$ or $2^{q}(0 \leqq q \leqq s-3)$. When $j=2^{q}, B$ is nonzero if and only if $i \leqq 2^{q}-1 . \quad A=\left(\begin{array}{c}2^{r-1}-1+t \\ 2^{q+1}+2 t\end{array}\right)$ is nonzero if and only if $t=0$. But this is impossible since then we have $t+i \leqq 2^{q}-1$ which contradicts to $t+i=2^{s-2}-2^{q}$. When $j=0$ we have $t+\imath=2^{s-2}$. $B$ is always nonzero. $A=\left(\begin{array}{c}2^{r-1}-1+t \\ 2 t\end{array}\right)$ is nonzero if and only if $t=2^{r-1}-2^{d}$ with $d \leqq r-1$. If $d<r-1$ then we have contradiction to the allowability. Therefore $d=r-1$ and $t=0$. Lastly we must check that $u$ is not contained in $\Sigma^{\prime \prime}$. Suppose $u$ is contained in $\Sigma^{\prime \prime}$ then we have $k=2^{r-1}-1$. $E$ is nonzero if and only if $t+i+j=2^{r-1}+2^{s-2}-2^{c}(c \leqq r-2)$. But this contradicts to the allowability since $i \leqq 2^{s-2}$ and $j \leqq 2^{s-3}$.

LEMMA 3.5. Let $r \geqq s \geqq 2$ and suppose that $Q^{a} Q^{b}(b>0)$ is allowable. Then $S q_{*}^{2^{r-1}} Q^{a} Q^{b}[1]$ contains $Q^{2^{r-2}+2^{s-1}-1} Q^{2^{r-2}+2^{s-2}-1}[1]$ if and only if $a=2^{r-1}+2^{s-1}-1$ and $b=2^{r-1}+2^{s-2}-1$.

Proof. We shall only prove the case when $r>s$. The case $r=s$ can be shown similarly. We shall again divide the proof into several cases. As in the proof of the preceding lemma, we shall put $u=Q^{2 r-2+2^{s-1}-1} Q^{2^{r-2}+2^{s-2-1}}[1]$ for brevity of notation. Also we may put $a=2^{r-1}+2^{s-1}-1+t$ and $b=2^{r-1}+2^{s-2}$ $-1-t$ for some $t$. Then the allowability of $Q^{a} Q^{b}$ implies that $3 t \leqq 2^{r-1}-1$. From the Adem and Nishida relations we have

$$
S q_{*}^{2^{r-1}} Q^{a} Q^{b}[1]=\Sigma^{\prime} A B Q^{x} Q^{y}[1]+\Sigma^{\prime \prime} A B C Q^{2^{r-1}+2^{s-1}+2^{s-2-2-j}} Q^{j}[1]
$$

where

$$
\begin{aligned}
& A=\left(\begin{array}{c}
2^{s-1}-1+t \\
2^{r-1}-2 i
\end{array}\right), \quad B=\left(\begin{array}{c}
2^{r-1}+2^{s-2}-1-(t+i) \\
i
\end{array}\right), \\
& C=\left(\begin{array}{c}
t+i+j-2^{r-1}-2^{s-2} \\
2 j-2^{s-1}+1-(t+i)
\end{array}\right), \\
& x=2^{s-1}-1+t+i \text { and } y=2^{r-1}+2^{s-2}-1-(t+i) .
\end{aligned}
$$

Here the first summation $\Sigma^{\prime}$ is over $i$ satisfying $3(t+i) \leqq 2^{r}-1$ and the second 
summation $\Sigma^{\prime \prime}$ is over $i, j$ such that $3(t+i) \geqq 2^{r}$ and $2 j \geqq 2^{s-1}-1+t+\imath$.

Case $s=2$ : If $\Sigma^{\prime}$ contains $u=Q^{2^{r-2}+1} Q^{2^{r-2}}[1]$, then we must have $t+i=2^{r-2}$ for some $i$ and $B=\left(\begin{array}{c}2^{r-2} \\ i\end{array}\right)$ is nonzero only if $i=0$ or $2^{r-2}$. But $i=0$ contradicts to the allowability. Hence we get $i=2^{r-2}, t=0$ and then $A$ is surely non-zero. This happens if and only if $a=2^{r-1}+1$ and $b=2^{r-1}$. Secondly if the term $u$ appears in $\Sigma^{\prime \prime}$, we must have $j=2^{r-2}$. Then $C=\left(\begin{array}{c}t+i-\left(2^{r-2}+1\right) \\ 2^{r-1}-1-(t+i)\end{array}\right)$ is nonzero if and only if $t+i=2^{r-1}-2^{q}(q \leqq r-3)$ by Lemma $3.3(2)$. Then $B=\left(\begin{array}{c}2^{r-1}-(t+i) \\ \imath\end{array}\right)$ is nonzero only if $i \leqq 2^{q}$. This contradicts to the allowability since then we have $t=2^{r-1}-2^{q}-i \geqq 2^{r-1}-2^{q+1} \geqq 2^{r-1}-2^{r-2}=2^{r-2}$. Thus $u$ does not appear in $\Sigma^{\prime \prime}$. Case $s>2$ : We first consider the case when $\Sigma^{\prime}$ contains $u$ as the term $A B Q^{x} Q^{y}[1]$. Then we must have $t+i=2^{r-2} . \quad B=\left(\begin{array}{c}2^{r-2}+2^{s-2}-1 \\ \imath\end{array}\right)$ is nonzero if and only if $0 \leqq i \leqq 2^{s-2}-1$ (i.e. $2^{r-2}-2^{s-2}+1 \leqq t \leqq 2^{r-2}$ ) or $i=2^{r-2}$ (i.e. $t=0$ ). By Lemma 3.3 (1), $A=\left(2^{s-1}-1+t\right)$ is nonzero if and only if $t=2^{s-1}-2^{q}(q \leqq s-1)$. Suppose $2^{r-2}-2^{s-2}+1 \leqq t \leqq 2^{r-2}$, then we have $s=r-1$ and $q \leqq r-4$. But then we have $t \geqq 2^{r-3}+2^{r-4}$. This contradicts to the allowability. Therefore we must have $t=0$ (i.e. $i=2^{r-2}$ ), and then $A$ is nonzero. We shall next see that $\Sigma^{\prime \prime}$ does not contain $u$. If $u$ is contained in $\Sigma^{\prime \prime}$, then we must have $j=2^{r-2}+2^{s-2}-1$ and by Lemma $3.3(2), C=\left(\begin{array}{c}t+i-\left(2^{r-2}+1\right) \\ 2^{r-1}-1-(t+i)\end{array}\right)$ is nonzero if and only if $t+\imath=2^{r-1}-2^{c}$ $(c \leqq r-3)$. But $A \neq 0$ implies that $i \leqq 2^{r-2}$. If $c \leqq r-4$ then we have $t \geqq 2^{r-3}+2^{r-4}$. This contradicts to the allowability. Therefore we have $c=r-3, t+i=2^{r-2}+2^{r-3}$, $B=\left(\begin{array}{c}2^{r-3}+2^{s-2}-1 \\ i\end{array}\right)$ and $A=\left(\begin{array}{c}2^{s-1}-1+t \\ 2 t-2^{r-2}\end{array}\right)$. We shall divide the case $s>2$ into three cases and see that in each case, $\Sigma^{\prime \prime}$ does not contain $u$.

Case $r-1=s>2$ : We find that $B$ is nonzero if and only if $i \leqq 2^{r-2}-1 . A$ is nonzero if and only if $t=2^{r-2}-2^{p}(p \leqq r-3)$ from a slightly modified version of Lemma 3.3 (1). If $p \leqq r-4$ then we get contradiction to the allowability. But when $p=r-3$, we have $i=2^{r-2}$ and $B$ vanishes.

Case $r-2=s>2: A$ is nonzero if and only if $t=2^{r-2}+2^{r-3}-2^{p}(p \leqq r-2)$ from Lemma 3.3 (1). And when $p<r-2$, we have contradiction to the allowability. Thus we have $t=2^{r-3}$ and $\imath=2^{r-2}$. In this case $B=\left(\begin{array}{c}2^{r-3}+2^{r-4}-1 \\ 2^{r-2}\end{array}\right)$ vanishes.

Case $r-3 \geqq s>2: A$ is nonzero if and only if $t=2^{r-3}+2^{s-1}-2^{p}(p \leqq s-1)$. Then we have $i=2^{r-2}-2^{s-1}+2^{p} \geqq 2^{r-2}-2^{r-4}>2^{r-3}+2^{s-2}-1$. Therefore $B$ vanishes.

LEMMA 3.6. Let $Q^{a} Q^{b}$ be allowable $(b>0)$ and $r>s \geqq 2$. Then the following two statements are equivalent.

(A) $S q_{*}^{2^{s-2}} S q_{*}^{2^{s-1}} \cdots S q_{*}^{2^{r-1}} Q^{a} Q^{b}[1]$ contains $Q^{2^{s-1}-1} Q^{2^{s-1}-1}[1]$.

(B) $S q_{*}^{2^{s-2}} S q_{*}^{2^{s-1}} Q^{a} Q^{b}[1]$ contains $Q^{2^{r-1-1}} Q^{2^{r-1}-1}[1]$.

Proof. Consider the following auxiliary statements : 
(C) $S q_{*}^{2 s} S q_{*}^{2^{s+1}} \cdots S q_{*}^{2 r-1} Q^{a} Q^{b}[1]$ contains $Q^{2^{s-1}} Q^{2^{s-1}+2^{s-2-1}}[1]$.

(D) $S q_{*}^{2^{t}} S q_{*}^{2^{t+1}} \cdots S q_{*}^{2^{r-1}} Q^{a} Q^{b}[1]$ contains $Q^{2^{t-1}+2^{s-1}-1} Q^{2^{t-1}+2^{s-2}-1}[1](s \leqq t \leqq r-1)$

(E) $a=2^{r-1}+2^{s-1}-1$ and $b=2^{r-1}+2^{s-2}-1$.

By Lemma 3.4, (A) is equivalent to (C). (C) is nothing but $\left(\mathrm{D}_{s}\right)$. Then by Lemma 3.5, $\left(D_{s}\right)$ is equivalent to $\left(D_{t}\right)$ for all $t .\left(D_{r-1}\right)$ is equivalent to $(E)$ by Lemma 3.5. Again by Lemma 3.4, (E) is equivalent to (B).

Having established these preparations we are now in a position to prove Theorem A.

\section{Proof of Theorem A:}

The smooth Kervaire class $k_{2 \imath}$ is primitive and so is the class $\operatorname{Sq}^{J}\left(k_{2 \imath}\right)$. So in order to prove the assertion of Theorem A, we have only to check that under the Kronecker pairing with the Pontrjagin ring generators of $H_{*}(S G)$,

$$
S q^{2^{2-1}} \cdots S q^{2^{s-1}} S q^{2^{s-2}}\left(k_{2 s-2}\right)
$$

and

$$
S q^{2 s-1} S q^{2 s-2}\left(k_{2 r-2}\right)
$$

have the same values. Suppose $I=\left(i_{1}, \cdots, i_{n}\right)$ is allowable and let $x=Q^{I}[1] *\left[1-2^{n}\right]$ be the Pontrjagin ring generator. When $n \neq 2,(1)$ and (2) have values 0 on $x$ by Proposition 2.8 , when we note that

$$
\begin{aligned}
\left\langle S q^{J}\left(k_{2 \imath-2}\right), Q^{I}[1] *\left[1-2^{n}\right]\right\rangle & =\left\langle k_{2 \imath-2},\left(S q^{J}\right)_{*} Q^{I}[1] *\left[1-2^{n}\right]\right\rangle \\
& =\sum_{j}\left\langle k_{2 \imath-2}, Q^{I j}[1] *\left[1-2^{n}\right]\right\rangle .
\end{aligned}
$$

Therefore Theorem A is proved if we can show that

$$
\begin{gathered}
\left\langle k_{2 s-2}, S q_{*}^{2^{s-2}} S q_{*}^{2^{s-1}} \cdots S q_{*}^{2^{r-1}} Q^{a} Q^{b}[1] *[-3]\right\rangle \\
=\left\langle k_{2 r-2}, S q_{*}^{2^{s-2}} S q_{*}^{2^{s-1}} Q^{a} Q^{b}[1] *[-3]\right\rangle,
\end{gathered}
$$

for all allowable $Q^{a} Q^{b}(b>0)$. But this is what we have shown in Lemma 2.7 and Lemma 3.6.

\section{§4. Proof of Theorem B.}

In order to apply the Rourke-Sullivan formula (1.1) for the Kervaire invariant, we have to compute the $W u$ classes of real projective spaces. To facilitate the calculations, we make the following definitions.

DEFINITION. Let $\varphi(t)=1+t+t^{2}+\cdots+t^{23}+\cdots$ be a formal power series with coefficients in $\boldsymbol{Z} / 2$. The coefficient of $t^{b}$ in the $a$-fold product $(\varphi(t))^{a}$ is denoted by $\{a, b\}$. When $a$ or $b$ is negative we set $\{a, b\}=0$ by convention. 
Definition. Let $N$ be a non-negative integer. Then $N$ has a unique expression

$$
N=2^{e_{1}}+2^{e_{2}}+\cdots+2^{e_{s}},
$$

with $0 \leqq e_{1}<e_{2}<\cdots<e_{s}$, which we call the ascending 2-adic expansion of $N$. When $N$ is not zero, $e_{1}$ the 2-order of $N$ is denoted by $\operatorname{ord}_{2}(N) . \quad$ If $N=0$, the ascending 2-adic expansion is vacuous $(s=0)$, and we set $\operatorname{ord}_{2}(0)=+\infty$.

Let $\eta$ be the Hopf line bundle over the real projective space $P^{N}$. Then the tangent bundle of $P^{N}$ is stably isomorphic to $(N+1) \eta$ and the total $W u$ class of $P^{N}$ is given by

$$
V\left(P^{N}\right)=(\varphi(x))^{N+1}=\sum_{\imath}\{N+1, i\} x^{2},
$$

where $x$ is the generator of $H^{1}\left(P^{N}\right)$.

We can easily prove the following lemma using the fact that the formal power series $\varphi(x)$ has the property $\varphi(x)^{2}=\varphi\left(x^{2}\right)$.

LEMMA 4.1. $\{a, b\}$ is characterized by the following properties $\{0,0\}=\{1,0\}=1, \quad\{2 a, 2 b\}=\{a, b\}, \quad\{2 a, 2 b+1\}=0, \quad\{2 a+1,2 b\}=\{a+1, b\}$ and $\{2 a+1,2 b+1\}=\{a, b\}$.

The lemma below gives a criterion for determining the value of $\{a, b\}$.

LEMMA 4.2. Let $a, b$ be positive and $b=2^{\imath_{1}}+2^{\imath_{2}}+\cdots+2^{\imath_{s}}$ be the ascending 2-adic expansion of $b$. Then $\{a, b\}$ is nonzero if and only if $a$ can be written as

$$
a=a_{1}+a_{2}+\cdots+a_{s}+a_{s+1},
$$

where $a_{1}, a_{2}, \cdots, a_{s+1}$ satisfy

$$
0<a_{\jmath} \leqq 2^{\imath} \jmath \text { and } a_{\jmath+1} \equiv 0 \bmod 2^{\imath \jmath+1} \quad(\jmath=1, \cdots, s) .
$$

In particular, if the 2-order of a is greater than that of $b$ then $\{a, b\}=0$ holds.

Proof. The proof proceeds by induction on $\mu(b)=\max \left\{i \mid 2^{2} \leqq b\right\}$. If $b$ is even $b=2 b^{\prime}\left(i_{1}>0\right)$, then we have $\{a, b\}=\left\{a^{\prime}+\varepsilon, b^{\prime}\right\}$ where $a=2 a^{\prime}+\varepsilon$ ( $\varepsilon=0$ or 1) by Lemma 4.1. By the inductive assumption, this is nonzero if and only if $a^{\prime}$ has an expression

$$
a^{\prime}+\varepsilon=a_{1}^{\prime}+\cdots+a_{s}^{\prime}+a_{s+1}^{\prime}
$$

such that

$$
0<a_{\jmath}^{\prime} \leqq 2^{2} \jmath^{-1} \text { and } a_{\jmath+1}^{\prime} \equiv 0 \bmod 2^{2} \jmath \quad(\jmath=1, \cdots, s),
$$

since $\mu\left(b^{\prime}\right)<\mu(b)$. Then we have the desired expression

$$
a=\left(2 a_{1}^{\prime}-\varepsilon\right)+2 a_{2}^{\prime}+\cdots+2 a_{s}^{\prime}+2 a_{s+1}^{\prime} .
$$


If $b$ is odd $b=2 b^{\prime}+1\left(i_{1}=0\right)$, then $\{a, b\}$ is nonzero only if $a$ is odd and in this case $\{a, b\}=\left\{(a-1) / 2, b^{\prime}\right\}$ by Lemma 4.1. This is nonzero if and only if there is an expression

$$
(a-1) / 2=a_{2}^{\prime}+\cdots+a_{s}^{\prime}+a_{s+1}^{\prime}
$$

with

$$
0<a_{\jmath}^{\prime} \leqq 2^{\imath^{-1}} \text { and } a_{j+1}^{\prime} \equiv 0 \bmod 2^{\imath}, \quad(j=2, \cdots, s),
$$

since $\mu\left(b^{\prime}\right)<\mu(b)$. Then we have the expression for $a$ :

$$
a=1+2 a_{2}^{\prime}+\cdots+2 a_{s}^{\prime}+2 a_{s+1}^{\prime},
$$

and the induction is complete.

Hereafter we shall assume that $n$ is a positive integer such that $n+1$ is not a power of 2 . Then $n+1$ has the ascending 2-adic expansion

$$
n+1=2^{e_{1}}+2^{e_{2}}+\cdots+2^{e_{s}} \quad(s \geqq 2) .
$$

Using the criterion of Lemma 4.2 , we can prove the following by elementary calculations.

LEMMA 4.3. $\left\{2 n+2-2^{\imath}, n+1-2^{j}\right\}$ is nonzero if and only if one of the following conditions is satisfied.

(1) $i<e_{1}$ and $j=i$ or $e_{1}$.

(2) $i=e_{1}$ and $j=e_{1}$ or $e_{2}$.

(3) $i=e_{s}+1$ and $j=e_{1}$.

Proof of Theorem $B$ :

Let $f: S^{2 m+1} \times P^{4 n-2 m+1} \rightarrow G / O$ be a smooth normal map and the smooth Kervaire class of dimension $2^{2}-2$ pulls back to

$$
f^{*}\left(k_{2 \imath-2}\right)=\delta_{i} 1 \otimes x^{2^{i}-2}+\varepsilon_{\imath} y \otimes x^{2^{2}-2 m-3}
$$

where $x \in H^{1}\left(P^{4 n-2 m+1}\right)$ and $y \in H^{2 m+1}\left(S^{2 m+1}\right)$ are generators. When $2^{2}-2>$ $4 n-2 m+1$ we set $\delta_{i}=0$ and when $2^{2}-2<2 m+1$ we set $\varepsilon_{\imath}=0$. Then by the Rourke-Sullivan formula (1.1), the Kervaire invariant for $f$ is given by

$$
c(f)=\sum_{i \geqq 2} \varepsilon_{i}\left\{2 n+1-m, n+1-2^{2-2}\right\} .
$$

We shall use the ascending 2-adic expansion (4.1) of $n+1$ in this proof. Let $e$ be the 2-order of $m+1$, then $m+1$ can be written $m+1=2^{e} m^{\prime}$ where $m^{\prime}$ is odd. Case $m^{\prime}=1$ : From (4.2) we have

$$
c(f)=\sum_{i \geqq 2} \varepsilon_{i}\left\{2 n+2-2^{e}, n+1-2^{2-2}\right\} .
$$

Then by Lemma 4.3 , we have 


$$
c(f)= \begin{cases}\varepsilon_{e+2}+\varepsilon_{e_{1}+2} & \text { if } e<e_{1}, \\ \varepsilon_{e_{1}+2}+\varepsilon_{e_{2}+2} & \text { if } e=e_{1}, \\ \varepsilon_{e_{1}+2} & \text { if } e=e_{s}+1 \text { and } \\ 0 & \text { otherwise. }\end{cases}
$$

If $e<e_{1}$, we have

$$
\begin{aligned}
& f^{*}\left(k_{a}\right)=\delta_{e+2}\left(1 \otimes x^{a}\right)+\varepsilon_{e+2}\left(y \otimes x^{a^{\prime}}\right), \\
& f^{*}\left(k_{b}\right)=\delta_{e_{1}+2}\left(1 \otimes x^{b}\right)+\varepsilon_{e_{1}+2}\left(y \otimes x^{b^{\prime}}\right),
\end{aligned}
$$

where $\quad a=2^{e+2}-2, \quad a^{\prime}=a-(2 m+1)=2^{e+1}-1, \quad b=2^{e_{1}+2}-2 \quad$ and $\quad b^{\prime}=b-(2 m+1)$ $=2^{e_{1}+2}-2^{e+1}-1$. By Theorem $\mathrm{A}$, we have

$$
S q^{2^{e_{1}+1}} \cdots S q^{2^{e+1}} S q^{2 e} f^{*}\left(k_{a}\right)=S q^{2^{e+1}} S q^{2 e} f^{*}\left(k_{b}\right) .
$$

This shows that $\varepsilon_{e+2}=\varepsilon_{e_{1}+2}$ and hence $c(f)$ vanishes.

If $e=e_{1}$, we can show that $\varepsilon_{e_{1}+2}=\varepsilon_{e_{2}+2}$ in a similar way. If $e=e_{s}+1$, then we have $\varepsilon_{e_{1}+2}=0$ since $2^{e_{1}+2}-2^{e+1}<0$. Thus the proof for $m^{\prime}=1$ is complete. Case $m^{\prime}>1$ : The Kervaire invariant is given by

$$
c(f)=\sum_{i \geqq 2} \varepsilon_{i}\left\{2 n+2-2^{e} m^{\prime}, n+1-2^{2-2}\right\},
$$

where $f^{*}\left(k_{2 \imath-2}\right)=\delta_{i} 1 \otimes x^{2^{2}-2}+\varepsilon_{\imath} y \otimes x^{2^{2}-2 m-3} . \quad \varepsilon_{\imath}$ vanishes for $2^{i}-2<2 m+1$. In particular, when $i=e+2$, we have $\varepsilon_{e+2}=0$ since $2^{e+2}<3 \cdot 2^{e+1} \leqq 2^{e+1} m^{\prime}=2 m+2$. And when $i>e+2$, by Theorem A, we have

$$
S q^{2 e+1} S q^{2 e} f^{*}\left(k_{2 \imath-2}\right)=S q^{2-1} \cdots S q^{2 e+1} S q^{2 e} f^{*}\left(k_{2 e+2-2}\right)=0 .
$$

On the other hand we have

$$
S q^{2^{e+1}} S q^{2^{e}} f^{*}\left(k_{2 \imath-2}\right)=\varepsilon_{i}\left(\begin{array}{c}
2^{\imath}-2 m+2^{e}-3 \\
2^{e+1}
\end{array}\right)\left(\begin{array}{c}
2^{\imath}-2 m-3 \\
2^{e}
\end{array}\right) y \otimes x^{c},
$$

where $c=2^{2}-2 m+2^{e+1}+2^{e}-3$. The two binomial coefficients in the above expression are nonzero since in the 2-adic expansion of $2^{2}-2 m-3$, the coefficient of $2^{e}$ is 1 and that of $2^{e+1}$ is zero. Thus the proof is complete if we can show that

$$
2^{i}-2+2^{e}+2^{e+1} \leqq 4 n+2 .
$$

First we consider the case when $2^{2}-2<2 n+1$. Then since $e \leqq i-3$, we have

$$
2^{\imath}-2+2^{e}+2^{e+1} \leqq 2^{\imath}-2+3 \cdot 2^{\imath-3} \leqq 2^{\imath+1}-2<4 n+2 .
$$

Secondly we assume that $2 n+1 \leqq 2^{2}-2 \leqq 4 n+2$, that is, $i=e_{s}+2$. In this case we find that $\left\{2 n+2-2^{e} m^{\prime}, n+1-2^{2-2}\right\}$ is nonzero only if $\operatorname{ord}_{2}\left(2 n+2-2^{e} m^{\prime}\right) \leqq e_{1}$. This means that $e \leqq e_{1}$. Then we have 


$$
\begin{aligned}
4 n+2-\left(2^{\imath}-2+2^{e}+2^{e+1}\right) & =4\left(n+1-2^{\imath-2}\right)-\left(2^{e}+2^{e+1}\right) \\
& \geqq 2^{e+2}-\left(2^{e}+2^{e+1}\right)=2^{e} \geqq 1 .
\end{aligned}
$$

\section{REFERENCES}

[1] G. Brumfiel, I. Madsen and R. J. Milgram, $P L$ characteristic classes and cobordism, Ann. of Math. 97 (1973), 82-159.

[2] M. Kato, Some problems in topology, "Manifolds Tokyo 1973", Proceedings of the international conference on manifolds and related topics in topology, Ed. by A. Hattori, 421-431, 1973.

[3] Y. KitADA, Orientation reversing involutions on Brieskorn spheres, Kodai Math. J. 9 (1986), 361-367.

[4] S. LOPEZ DE MEdRano, Involutions on manifolds, Springer, 1971.

[5] I. MAdsen, On the action of the Dyer-Lashof algebra in $H_{*}(G)$, Pac. Jour. Math. 60 (1975), 235-275.

[6] I. Madsen and R. J. Milgram, The classifying spaces for surgery and cobordism of manifolds, Ann. of Math. Studies, 92, Princeton Univ. Press, 1979.

[7] R. J. Milgram, The mod 2 spherical characteristic classes, Ann. of Math. 92 (1970), 238-261.

[8] G. NishidA, Cohomology operations in iterated loop spaces, Proc. Japan Acad. 44 (1968), 104-109.

[9] C.P. Rourke And D.P. Sullivan, On the Kervaire obstruction, Ann. of Math. 94 (1971), 397-413.

[10] N.E. Steenrod And D. B. A. Epstein, Cohomology operations, Annals of Math. Studies, 50, Princeton Univ. Press, 1962.

[11] C. T.C. W AlL, Surgery on compact manifolds, Academic Press, London, 1970.

Department of Applied Mathematics

FACULTY OF ENGINEERING

Yokohama National University

Tokiwadai, Hodogaya-ku, Yokohama

240, JAPAN 